\title{
Impact of radiator length in the emitted power for a high gain harmonic generation free-electron laser
}

\begin{abstract}
Enrico Allaria*
Elettra-Sincrotrone Trieste, Strada Statale 14, 34149 Basovizza, Trieste, Italy

(Received 19 April 2012; revised manuscript received 29 January 2013; published 12 March 2013)

We present a numerical study that characterizes the dependence on the radiator length of the output power produced by a free-electron laser (FEL) operated in the high gain harmonic generation (HGHG) configuration. Using the main parameters of the FERMI@Elettra FEL, numerical simulations of the FEL process have been performed for different lengths of the radiator. Our results show that in the case of HGHG the achievable output power has a dependence on the radiator length that is linear. The impact of the electron beam parameters on the achievable maximum power vs radiator length dependence is also studied. A normalization of the results to the FEL saturation power and to the FEL gain length shows that this dependence can be expressed by a universal linear equation that, in some conditions, is independent on the electron beam current and brightness. The reported results could be useful for the design of future FELs based on the HGHG scheme and could be used for a quick estimate of the best undulator length.

DOI: 10.1103/PhysRevSTAB.16.030703

PACS numbers: 41.60.Cr, 42.55.Vc
\end{abstract}

\section{INTRODUCTION}

Free-electron lasers (FELs) based on high brightness electron beams are becoming a key instrument in the recent development of science. High brightness and short pulses produced by FELs from vacuum ultraviolet (VUV) down to $\mathrm{x}$ ray are now available in several user facilities around the world [1-4] and more facilities are expected to begin operation over the next few years [5]. Between possible schemes that can be used to produce FEL pulses in the VUV-soft $x$ ray there is particular interest on high gain harmonic generation (HGHG) [6] that uses an external laser to initiate the process and can improve the FEL coherence and stability. The use of harmonic generation to produce FEL pulses at short wavelength starting from an external seed laser at long wavelength was originally proposed in [7] and was then applied to high gain single pass FELs to generate IR [8] and VUV coherent emission [9]. In the past decade, HGHG has been demonstrated and studied in several FEL test facilities [10-15] down to the VUV spectral range. More recently, HGHG has been demonstrated to efficiently work down to the extreme ultraviolet [4]. Although experiments have been done in different experimental conditions, including very different electron beam and undulator parameters, all experiments have shown the capability of HGHG to produce FEL pulses with a wellcontrolled and narrow bandwidth. Because of the quite different electron beam parameters and the length of used undulator lengths used in many of these experiments, the condition of strong exponential growth of the power along

\footnotetext{
*enrico.allaria@elettra.trieste.it
}

Published by the American Physical Society under the terms of the Creative Commons Attribution 3.0 License. Further distribution of this work must maintain attribution to the author( $s)$ and the published article's title, journal citation, and DOI. the undulator does not apply, especially in cases where the undulator length is less than a meter. In those cases, the FEL power only has coherent emission in the radiator without exponential gain and the process is generally called coherent harmonic generation (CHG). Strictly speaking, HGHG should generally be associated with cases showing true exponential growth of the power in a radiator whose length therefore must be at least a few FEL gain lengths. In this work we focus on the impact of the length for the final radiator in the case of a HGHG FEL using the parameters of FERMI as a case study.

Here we will show HGHG results for the functional dependence of output power upon undulator length are quite different from what occurs for a simple FEL amplifier that starts from a weak signal. In FEL amplifiers, whose input signal is provided by an external source or by the spontaneous emission as in self-amplified stimulated emission (SASE) $[16,17]$, the output power drastically depends on the length of the radiator. Indeed the power that a FEL amplifier can produce increases exponentially with the length of the available radiator until saturation is reached. In contrast, we will find that the optimized HGHG output power in the situation where there is plentiful seed power will grow linearly rather than exponentially with radiator length.

The paper is organized as follows. Section II introduces the HGHG mechanism and presents the parameters of FERMI considered for this study. Section. III reports the results of numerical simulations for one configuration and his analysis. More cases and a generalization of the results are finally discussed in Sec. IV. Conclusions are reported in Sec. V.

\section{HGHG MECHANISM AND FERMI FEL}

In the HGHG configuration the FEL process is initiated by an external laser. The process can be divided into three 
parts, the modulator, the bunching section, and the radiator. In the modulator the electron beam wiggling due to the magnetic field of the undulator is at resonance with the electromagnetic field of the external seed laser. Because of the interaction with the seed laser, the electron beam becomes energy modulated with a periodicity equal to the seed laser wavelength [6]. The energy modulation is then converted into density modulation when the electron beam passes through the bunching section. The bunching section is composed by a dispersive element where highenergy electrons perform a shorter path than low energy electrons. As a result, density modulation is created at the seed laser wavelength and also at its harmonics. In the case of FERMI a significant amount of bunching is expected up to the tenth harmonic [18] and good FEL performance has been already demonstrated up to the height [4]. The radiator is based on a long undulator which is set so that the electron beam is at resonance with the desired harmonic of the seed laser. Because the electron beam is already bunched at that wavelength, electrons immediately emit coherently. This coherent emission is several orders of magnitude larger than the spontaneous emission. In the case of CHG without significant FEL gain, the power initially grows approximately quadratically with undulator length; the growth rate eventually drops to zero either due to dynamical debunching effects and/or diffraction losses. In true $\mathrm{HGHG}$, further interaction inside the radiator between the electron beam and the produced coherent radiation leads to increased coherent bunching beyond the value produced by the bunching section and eventually an exponential increase of the FEL power with increasing radiator length. Two different regimes of power growth can be identified in a HGHG FEL. In the first part, where only coherent emission is generated, power increases quadratically with the undulator length while an exponential growth of the power along the undulator characterizes the second part [6]. In this final part, the bunching increase is associated with the self-induced effect that characterizes high gain FELs and SASE [19]. Like for FEL amplifiers and SASE, a well optimized HGHG is characterized by an exponential growth of the power along the last part of the radiator. However, at variance of SASE, in HGHG the power level before the amplification can be controlled by changing the seeding parameters. This opens the possibility to compensate a decreased output power associated with a limited radiator length by optimizing the seed power to the actual length. For this reason, we expect that the maximum power produced by HGHG should be less critically dependent on radiator length. This property is confirmed by our simulations presented in Sec. III.

\section{A. The FERMI free-electron laser}

FERMI is a free-electron laser user facility that has been designed on the HGHG configuration for producing high quality FEL pulses in the spectral range from $80 \mathrm{~nm}$ down to $4 \mathrm{~nm}$ [20]. The wide spectral range is covered by two different FEL lines that use the same electron beam. In this work we focus on the first FEL, FEL-1, designed for covering the $80-20 \mathrm{~nm}$ spectral range with single stage HGHG. FERMI uses electron beams accelerated by a normal conducting linear accelerator with electron beam energy in the range between 0.9 and $1.5 \mathrm{GeV}$. Electron bunches with a charge of few hundreds of $\mathrm{pC}$ are generated in a high brightness $\mathrm{rf}$ photocathode gun. The accelerator has two bunch compressors and has been designed to preserve the high quality and brightness of the beam up to the entrance of the undulator. A more detailed description of the accelerator design and configuration is reported in [21]. FEL-1 studies at FERMI over the past couple years have generated high quality FEL pulses in the 26 to $65 \mathrm{~nm}$ spectral range starting from an UV seed laser at about $260 \mathrm{~nm}[4,22]$. While the design of FERMI is based on an electron beam with $800 \mathrm{~A}$ of peak current [20], initial operations have used 350 and 500 A beams. For this work we focus on the $500 \mathrm{~A}$ case. Other electron beam parameters characterizing the FERMI electron beam used in this work are listed in Table I.

Because HGHG relies on the exponential amplification occurring in the radiator, FEL performance depends on the electron beam brightness and peak current. In order to confirm the generality of our findings, the 350 and $800 \mathrm{~A}$ cases have been also studied and results are summarized in Sec. IV.

The undulator parameters used for these studies are those of the first FEL at FERMI (FEL-1) [20] and the scheme is sketched in Fig. 1. The modulator (MOD) is a 3 meter long planar undulator with $100 \mathrm{~mm}$ undulator period. The dispersive section (R56) is a three dipole chicane whose dispersion can be set in the range $0-150 \mu \mathrm{m}$. The radiator (RAD) is composed of six undulators 2.4 meter long with a period of $55 \mathrm{~mm}$ and 1.2 meter break between them. Undulator optics allow an average electron beam size in the undulator of about $100 \mu \mathrm{m}$.

FERMI has the possibility to extend the length of the present radiator by adding two additional undulators. In order to study the effects of undulator length also beyond the present capability of FERMI we have considered a radiator up to ten undulators long. Since we are interested in the study of HGHG FELs our studies start from a minimum length for the radiator of three undulators.

TABLE I. Electron beam parameters used for this work and describing the parameters for FERMI electron beam.

\begin{tabular}{lcc}
\hline \hline Parameter & Value & Units \\
\hline Energy & 1.2 & $\mathrm{GeV}$ \\
Normalized transverse emittance & 1.0 & $\mathrm{~mm} \mathrm{mrad}$ \\
Energy spread & 100 & $\mathrm{keV}$ \\
Average beta & 10 & $\mathrm{~m}$ \\
Peak current & $350-500-800$ & $\mathrm{~A}$ \\
\hline \hline
\end{tabular}




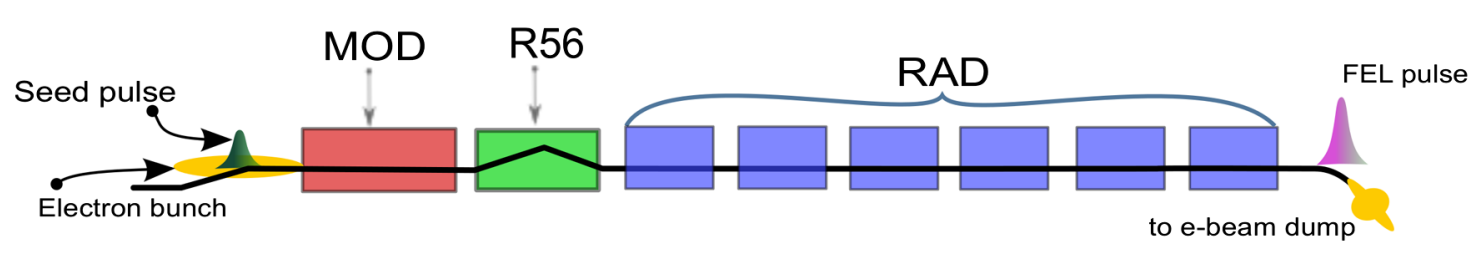

FIG. 1. Sketch of the undulators of FERMI FEL-1. The system counts a modulator (MOD), a dispersive section (R56), and six undulators define the final radiator (RAD). Between each undulator some diagnostic components, electron beam optics (quadrupoles) and phase shifter to properly set the interference between adjacent undulator are present.

Shorter radiators will only allow coherent emission and should be considered as CHG configurations.

The studied setup uses a 260-nm wavelength seed with a pulse length of 150 fs FWHM and a peak power that for this work is varied in the range between $20 \mathrm{MW}$ and $1 \mathrm{GW}$. The seed power level is used as an optimization parameter to maximize the FEL output power for the various cases. The dispersive section strength parameter R56 is also used as an optimization parameter. In this section the energy modulation produced by the external seed is converted to a strong density modulation and it is obvious that stronger seed powers will be in general accompanied by smaller R56 values for optimal performance in the downstream radiator. In our study the final radiator is tuned to $20 \mathrm{~nm}$ (the 13th harmonic of the seed laser) that is the shorter wavelength for FEL-1 and the more sensitive to the quality of the electron beam.

\section{RESULTS}

In order to study the effects of radiator length, we performed series of numerical simulations of the FEL process. Being interested mainly in the performance in terms of FEL power and not the spectral and temporal structures of the FEL pulses, we performed "timeindependent" single slice simulations with the FEL code GINGER [23]. The use of time-independent simulations is possible in the case of FERMI because of the relatively long pulse of the seed laser compared to the slippage length over the radiator. Moreover, in the case of FERMI, where the seeding pulse is much shorter than the electron beam, the electron beam can also be considered uniform. For these reasons the temporal effects like superradiance $[19,24]$ can be considered to have a negligible impact. For some cases, time-dependent simulations have been performed and the results, not presented here, are in good agreement with time-independent predictions.

First indications of the FEL characteristics can be obtained by using the Xie formula [25] with electron and undulator parameters. The Xie formula provides an estimation of the achievable performance for the FEL used as an amplifier at the desired wavelength. It is possible to calculate the expected FEL gain length, the expected output power given the length of the undulator, and also the expected maximum output power at which the FEL would saturate in case of a long enough undulator. Although this formalism has been defined for simple FEL amplifiers it can be adapted to give some information also to the case of the HGHG FEL. In order to estimate the gain and the expected saturation power in a HGHG FEL with the Xie formula, it is necessary to consider that the electron beam entering into the radiator has an energy spread that has been partially spoiled by the seeding process. In the case reported here, we have assumed that the energy spread of the electron beam entering into the radiator for the HGHG case is about $1 \mathrm{MeV}$ which is a factor 10 larger than the value used for the simple amplifier and reported in Table I. Such an increase of the energy spread is the result of the seeding necessary to produce the bunching. Using this increased energy spread, we can have an estimation of the gain length that will characterize the FEL in the second part of the radiator. The calculated values for the gain length and saturation power at $20 \mathrm{~nm}$ for the cases studied here are reported in Table II.

\section{A. Radiation growth in FEL amplifier and HGHG}

Because of the different processes that originate the input emission the optimization of FELs operating in the HGHG regime is very different with respect to the optimization of SASE amplifier or SASE. For an amplifier FEL or a SASE the input signal is defined by the available power from the seeding source or by the shot noise of the electron beam and these are given quantities that we cannot increase at will. As a consequence there are no parameters to control the startup of the FEL process. On the contrary, in an HGHG the startup can be optimized by controlling the seeding process. HGHG, indeed, uses a seed that occurs at wavelengths that are much longer than the final FEL

TABLE II. Calculated FEL parameters at $20 \mathrm{~nm}$, gain length, saturation power, and saturation length using the Xie formula [25] with electron and undulator parameters used for our studies.

\begin{tabular}{lccc}
\hline \hline & $350 \mathrm{~A}$ & $500 \mathrm{~A}$ & $800 \mathrm{~A}$ \\
\hline SASE gain length $(\mathrm{m})$ & 1.9 & 1.6 & 1.3 \\
HGHG gain length $(\mathrm{m})$ & 2.6 & 2.0 & 1.6 \\
Saturation power $(\mathrm{GW})$ & 0.65 & 1.1 & 2.3 \\
SASE saturation length $(\mathrm{m})^{\mathrm{a}}$ & 53 & 47 & 40 \\
FEL $\rho$ & $1.9 \times 10^{-3}$ & $2.1 \times 10^{-3}$ & $2.5 \times 10^{-3}$ \\
\hline \hline
\end{tabular}

${ }^{\text {a The length estimated here takes into account the breaks between }}$ the undulators of FERMI. 
wavelength. Usually the seeding source is a laser in the UV and there is a significant margin in the intensity one can use. This is not the case if HGHG is initiated by other sources like high harmonics generated in gas that may have limits in the available power [12]. In this work we consider the use of a UV laser as a seed that essentially has no limitation in the available power as it is for FERMI.

In the case of an amplifier, the radiator has to be long enough to allow the FEL to reach saturation while starting with the available input power. For SASE configurations that begin with a virtual signal from shot noise, typical 20 gain lengths are required for saturation. In the case of a shorter undulator the FEL power would be significantly reduced. On the contrary in the HGHG case, since the amount of bunching and coherent emission that initiate the process can be optimized, the dependence of the FEL output power on the length of the radiator is less critical. In the case of a shorter undulator, a stronger seed can be used that produces higher bunching and consequently a stronger coherent signal in the first part of the radiator. Such an increase of the initial coherent signal can partially compensate for the shorter undulator.

The difference between a simple, seeded FEL amplifier and HGHG is evident in Fig. 2. Here we report the results of simulations for three different cases of HGHG with different radiator lengths and an amplifier initiated by a weak signal comparable to the shot noise expected for a
SASE. All cases use the same electron beam (whose main parameter are described in Table I, $500 \mathrm{~A}$ ); the three HGHG cases have been optimized by choosing the best combination of seed power and dispersive section strength that maximize the output power.

From the results reported in Fig. 2 it is clear that, although the power increases exponentially along the radiator also in the case of HGHG [Fig. 2(b)], the final power shows a smaller dependence on the available length for the radiator. Indeed, as a consequence of the different optimization, the maximum power produced from a radiator with six undulators is larger than the power produced from the sixth undulator when the FEL has been optimized for maximizing the power coming out from the seventh undulator.

This weaker dependence of power in HGHG on the radiator length is a consequence of the fact that the seeding and bunching process can be optimized in order to allow the FEL to reach the maximum bunching close to the end of the available radiator [Fig. 2(c)] independently of the available undulator length. By controlling the seeding process (specifically, the seed power), it is generally possible to increase the initial bunching to a value that results in the coherent bunching at the desired harmonic to reach a maximum before the electron beam exits the radiator. However, increasing the bunching has a price: higher bunching requires a stronger seed that is also responsible
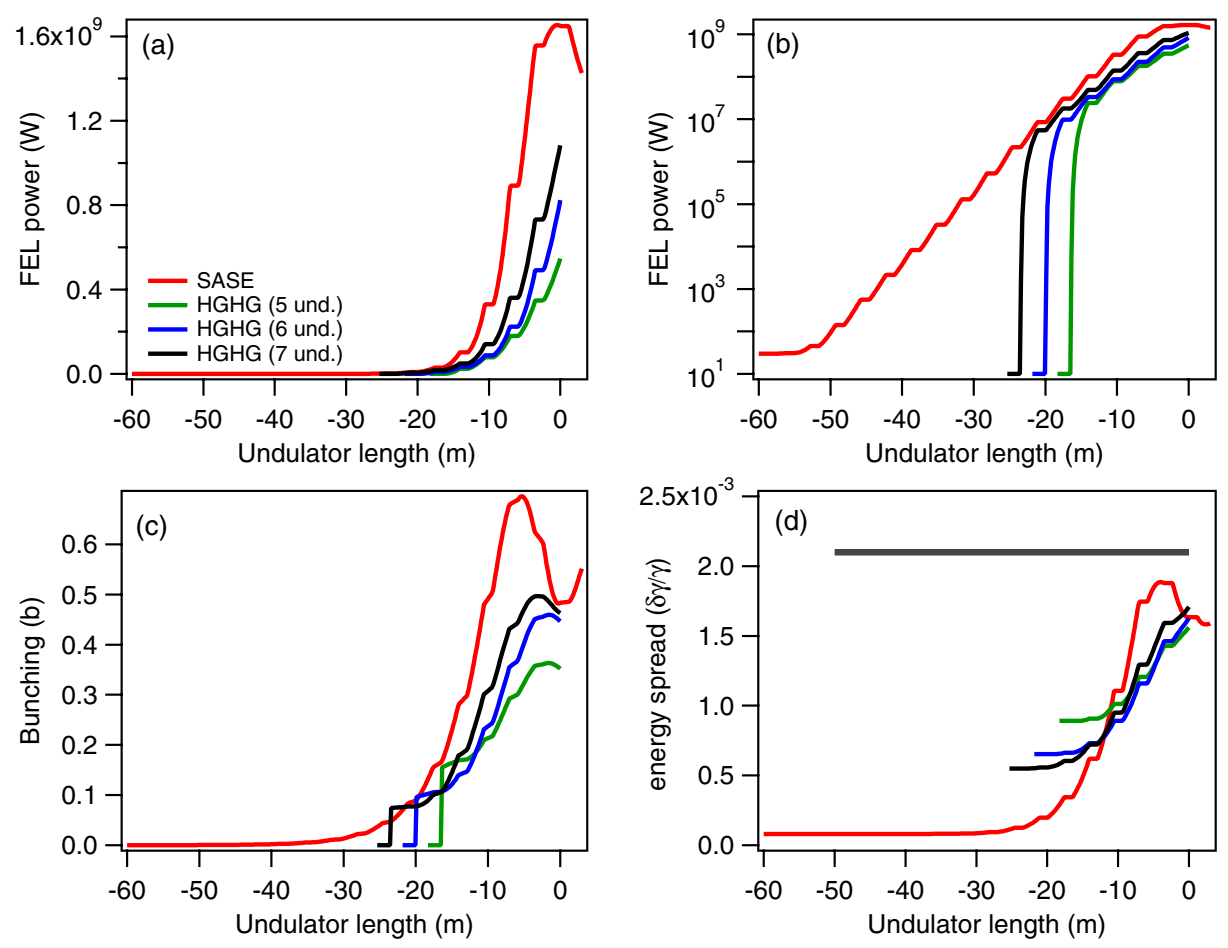

FIG. 2. FEL evolution as a function of radiator length in an amplifier (red) and HGHG with five, six, and seven undulators (green, blue, grey, respectively). FEL power is reported in linear (a) and logarithmic (b) scales, showing a slightly larger gain length for HGHG cases. In all cases the bunching (c) shows a local maximum around the last undulator and relative energy spread (d) that approaches the FEL $\rho$ parameter. 
for a larger energy spread of the beam entering in the radiator. The larger energy spread is responsible for a less efficient FEL process that leads to a weaker amplification and a smaller absolute value for the maximum bunching and of the maximum power. A detailed analysis of this mechanism is reported in the next section.

\section{B. HGHG optimizations for different radiator lengths}

We report here the results for the maximal achievable power at $20 \mathrm{~nm}$ for various radiator lengths that have been obtained using the nominal electron beam parameters [Table I (500 A)]. For this electron beam the gain length for the HGHG is estimated using the Xie equations to be about $2.0 \mathrm{~m}$ (Table II). By varying the number of undulator in the final radiator between 3 and 10 we are exploring cases where the number of gain lengths goes from about 4 to 12. For short radiators, we are in a condition that allows only marginal FEL gain and the radiation is mainly coherent emission from a prebunched beam. The long radiator case allows, instead, a significant gain of the power in the undulator requiring a small initial bunching and a weaker seed laser. As already anticipated, a minimum of three undulators has been considered since we are interested in the HGHG configuration that require at least few gain lengths to develop.

For each radiator length the FEL has been optimized with two dimensional scans over seeding power and dispersive section strength (R56) with a goal maximum FEL output power.

Results of the optimization are reported in Fig. 3. Figure 3(a) shows the increase of the maximum output power that can be generated as the length of the radiator is varied from about 10 meters (three undulators) up to 35 meters (ten undulators). It is important to note here that the power HGHG can produce increases almost linearly with the length of the radiator. This is the result of different seeding configurations that have been used for each undulator length. In Fig. 3(b) the optimal values for
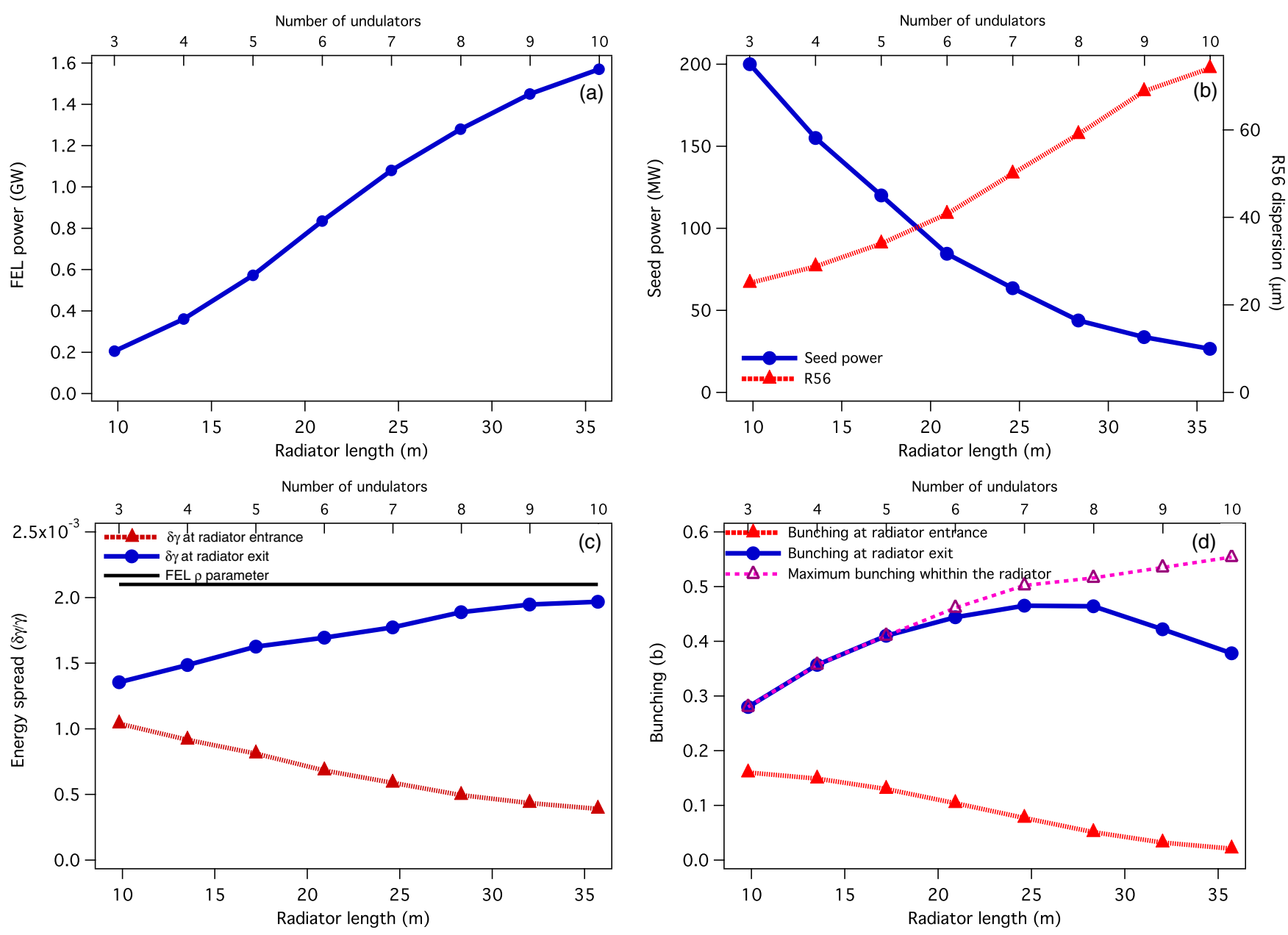

FIG. 3. (a) Maximum power obtained from HGHG at $20 \mathrm{~nm}$ using the electron beam with $500 \mathrm{~A}$ of peak current as a function of the number of undulators used for the final radiator. (b) Optimized values for seeding power (blue) and dispersive section strength (red) for maximizing the output power for each radiator length. (c) Normalized energy spread of the beam at the radiator entrance (red) and exit (blue), for comparison the FEL $\rho$ parameter is also plotted. (d) Bunching of the electron beam at the FEL wavelength at the radiator entrance (red) and exit (blue); the maximum value of the bunching achieved within the radiator (purple) is also plotted. 
the seed laser power and dispersive section as a function of the number of undulators are plotted. The results clearly show how, in the case of a short radiator, a quite strong seed is needed in order to produce a good bunching in the beam entering the radiator [Fig. 3(d), red curve]. Because the strong seeding the beam has also a quite high-energy spread [Fig. 3(c), red curve]. Large energy spread together with a short undulator does not allow a significant FEL growth. The fact that, for short radiators, the energy spread and the bunching at the exit of the radiator are only slightly larger than the one at the entrance are indicating that the produced FEL emission comes mainly from the coherent emission from a prebunched beam. As the length of the radiator increases, there is more margin for gain and the input bunching and seed laser can be reduced allowing a more efficient FEL growth.

It is also worth noting that starting from a radiator with seven undulators the maximum bunching is produced by the FEL process before the end of the radiator [Fig. 3(d)]. A radiator with seven undulators is also the one that allows the FEL operated in HGHG to produce an output power which is close to the saturation power predicted by Xie equations (Table II).

\section{NORMALIZED RESULTS}

In this section results for the maximum power as a function of the radiator length are presented for three electron beams characterized by different values for the peak current. The case studied in the previous section using an electron beam with $500 \mathrm{~A}$ of peak current is here compared with 350 and 800 A beams. Other electron beam parameters remain the same as reported in Table I. Similarly to what has been done in the previous section also for the 350 and $800 \mathrm{~A}$ beams, the seeding has been optimized in order to have the maximum power for each radiator length. In order to better compare the results from different electron beams we use a normalization. For each value of the electron beam current the obtained power is normalized to the corresponding saturation power calculated with the Xie formula and reported in Table II. Similarly the length of the radiator is normalized to the corresponding gain lengths also reported in Table II. The results of the numerical simulations for the three cases are plotted on the same graph in Fig. 4.

Data reported in Fig. 4 confirm the results of Fig. 3(a) showing that in the case of HGHG the maximum FEL power that can be produced from a given radiator only increases linearly with the length of the radiator also for electron beams characterized by higher and lower peak current. Moreover, Fig. 4 shows that in the particular case of FERMI and with the normalization adopted here, the ratio between FEL power and radiator length is independent on the electron beam current; we can expect this to be valid until the gain length is shorter than the Rayleigh length of the produced radiation. This

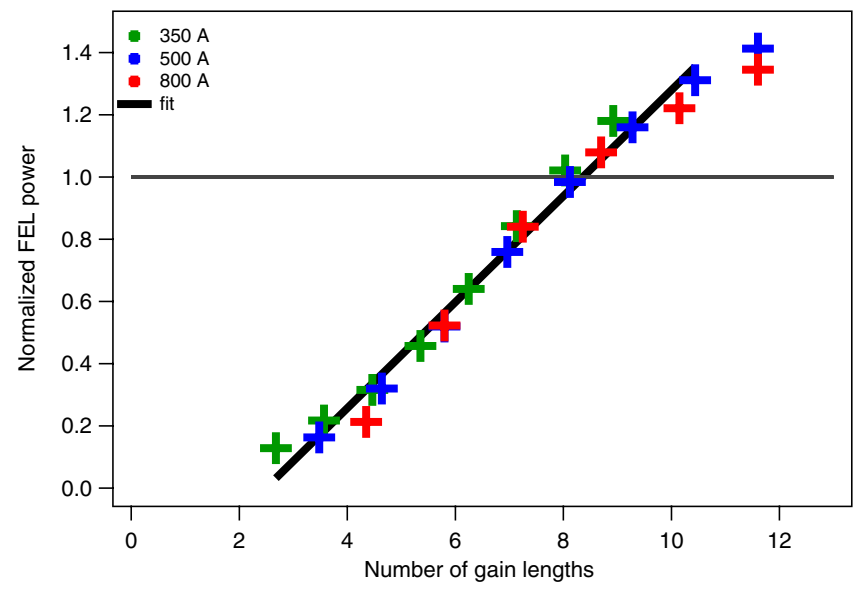

FIG. 4. Maximum power normalized to the saturation power for HGHG with three different values for the beam current, 350, 500, and 800 A (green, blue, and red, respectively). Normalized FEL power is reported as a function of the length of the radiator normalized to the FEL gain length.

allows us to define a universal scaling law for the maximum FEL power as a function of the radiator length. A linear fit of the results using the data associated to a gain length between 3 and 10 for the three current cases gives the following equation:

$$
\frac{P_{\mathrm{HGHG}}}{P_{\mathrm{SAT}}}=0.17\left(\frac{L}{L_{g}}-2.5\right)
$$

Equation (1) describes the expected power from HGHG $\left(P_{\mathrm{HGHG}}\right)$ normalized to the saturation power $P_{\mathrm{SAT}}$, where $L$ is the length of the radiator, $L_{g}$ and $P_{\mathrm{SAT}}$ the gain length and saturation power calculated with Xie. Equation (1) can be used to predict the expected power from an FEL in HGHG configuration using a given undulator whose length is larger than three gain lengths and shorter than ten gain lengths. This corresponds to the typical range of radiator length considered for HGHG systems. Additional studies show that the same behavior of the normalized power vs normalized undulator length is obtained using electron beam with slightly different values for the emittance and energy spread. This suggests that, provided the beam is bright enough to generate FEL radiation, the relation reported in Eq. (1) is independent on the beam brightness. It is important to point out that the validity of Eq. (1) is limited to an HGHG operated in a condition similar to the one here described where both the seed laser power and the dispersive section strength can be changed freely.

Reported results summarized by Eq. (1) could be useful in the design of the FEL facility based on HGHG. This simple equation can give a quick estimation of the undulator length in the FEL performance and guide for the decision of the length for the radiator length with all the constraints (space, budget, etc.). 


\section{CONCLUSIONS}

We reported about a study that shows the linear dependence of the FEL power that can be produced by an FEL in the HGHG configuration as a function of the length of the available radiator. While for a given radiator length the power will still have quadratic or exponential growth along the undulator length, the final power that an HGHG FEL can produce depends only linearly with the length of the radiator. This characteristic of HGHG is associated to the possibility to change the seeding parameters in order to optimize the bunching of the beam entering into the radiator according to the radiator length. Using the standard FEL normalization a universal linear dependence of power as a function of the length has been found. The work has been focused on the effect of the length of the radiation in the power considering electron beam characterized by different current, additional studies show that the same linear dependence is valid also for electron beam with varying emittance and/or energy spread.

\section{ACKNOWLEDGMENTS}

The author acknowledges the FERMI team for useful discussions and is particularly in debt with G. De Ninno and W. Fawley for inspiring discussions.

[1] W. Ackermann et al., Nat. Photonics 1, 336 (2007).

[2] P. Emma et al., Nature Photon. 4, 641 (2010).

[3] T. Ishikawa et al., Nature Photon. 6, 540 (2012).

[4] E. Allaria et al., Nature Photon. 6, 699 (2012).

[5] B. McNeil and N. Thompson, Nature Photon. 4, 814 (2010).

[6] L. Yu, Phys. Rev. A 44, 5178 (1991).
[7] B. Girard, Y. Lapierre, J. Ortega, C. Bazin, M. Billardon, P. Elleaume, M. Bergher, M. Velghe, and Y. Petroff, Phys. Rev. Lett. 53, 2405 (1984).

[8] L. Yu et al., Science 289, 932 (2000).

[9] L. Yu et al., Phys. Rev. Lett. 91, 074801 (2003).

[10] M. Labat, G. Lambert, M. E. Couprie, M. Shimada, M. Katoh, M. Hosaka, Y. Takashima, T. Hara, and A. Mochihashi, Nucl. Instrum. Methods Phys. Res., Sect. A 593, 1 (2008).

[11] G. DeNinno et al., Phys. Rev. Lett. 101, 053902 (2008).

[12] M. Labat et al., Phys. Rev. Lett. 107, 224801 (2011).

[13] D. Xiang et al., Phys. Rev. Lett. 105, 114801 (2010).

[14] N. Cutic, F. Lindau, S. Thorin, S. Werin, J. Bahrdt, W. Eberhardt, K. Holldack, C. Erny, A. L'Huillier, and E. Mansten, Phys. Rev. ST Accel. Beams 14, 030706 (2011).

[15] C. Feng, T. Zhang, J. Chen, H. Deng, M. Zhang, X. Wang, B. Liu, T. Lan, D. Wang, and Z. Zhao, Phys. Rev. ST Accel. Beams 14, 090701 (2011).

[16] A. Kondratenko and E. Saldin, Part. Accel. 10, 207 (1980) [http://cds.cern.ch/record/1107977/files/p207.pdf].

[17] R. Bonifacio, C. Pellegrini, and L. Narducci, Opt. Commun. 50, 373 (1984).

[18] E. Allaria and G. DeNinno, Phys. Rev. Lett. 99, 014801 (2007).

[19] R. Bonifacio et al., Riv. Nuovo Cimento 13, 1 (1990).

[20] C. Bocchetta et al., FERMI@Elettra FEL Conceptual Design Report No. ST/F-TN-07/12, 2007.

[21] S. DiMitri et al., in Proceedings of FEL2010, Malmö, Sweden (SPIE, Bellingham, WA, 2010), THPB03.

[22] S. DiMitri et al., in Proceedings of the International Society for Optical Engineering, Prague, Czechoslovakia (SPIE, Bellingham, WA, 2011), p. 8078.

[23] W. Fawley, Lawrence Berkeley National Laboratory, Report No. lbnl-49625, 2002.

[24] L. Giannessi, P. Musumeci, and S. Spampinati, J. Appl. Phys. 98, 043110 (2005).

[25] M. Xie, Nucl. Instrum. Methods Phys. Res., Sect. A 445, 59 (2000). 